\title{
Wide-bandwidth charge sensitivity with a radio-frequency field-effect transistor
}

\author{
Katsuhiko Nishiguchi, ${ }^{1, a)}$ Hiroshi Yamaguchi, ${ }^{1}$ Akira Fujiwara, ${ }^{1}$ Herre S. J. van der Zant, ${ }^{2}$ \\ and Gary A. Steele ${ }^{2}$ \\ ${ }^{1}$ NTT Basic Research Laboratories, Nippon Telegraph and Telephone Corporation, \\ 3-1 Morinosato Wakamiya, Atsugi, Kanagawa 243-0198, Japan \\ ${ }^{2}$ Kavli Institute of Nanoscience, Delft University of Technology, Lorentzweg 1, 2628CJ Delft, The Netherlands
}

(Received 19 April 2013; accepted 11 September 2013; published online 30 September 2013)

\begin{abstract}
We demonstrate high-speed charge detection at room temperature with single-electron resolution by using a radio-frequency field-effect transistor (RF-FET). The RF-FET combines a nanometerscale silicon FET with an impedance-matching circuit composed of an inductor and capacitor. Driving the RF-FET with a carrier signal at its resonance frequency, small signals at the transistor's gate modulate the impedance of the resonant circuit, which is monitored at high speed using the reflected signal. The RF-FET driven by high-power carrier signals enables a charge sensitivity of $2 \times 10^{-4} \mathrm{e} / \mathrm{Hz}^{0.5}$ at a readout bandwidth of $20 \mathrm{MHz}$. (C) 2013 AIP Publishing LLC. [http://dx.doi.org/10.1063/1.4822430]
\end{abstract}

Charge sensors based on a nanometer-sale transistor have been widely studied to detect extremely small signals, ${ }^{1}$ such as image charges from DNA, ${ }^{2}$ single-electron chargecoupled devices, ${ }^{3}$ and mechanical motion. ${ }^{4}$ One of the major breakthroughs in charge sensors was the development of the radio-frequency single-electron transistor (RF-SET). By using an impedance matching circuit, the RF-SET achieved both extremely high charge sensitivity and a wide bandwidth $\left(1.2 \times 10^{-5} \mathrm{e} / \mathrm{Hz}^{0.5}\right.$ at $\left.1.1 \mathrm{MHz}\right) .^{5}$ Because of this unique combination, the RF-SET also has stimulated fundamental research on single-electron transport via a quantum dot for quantum information processing. ${ }^{6-8}$ The RF-SET offers three advantages for charge detection: (i) a single-electron transistor with high charge sensitivity due to Coulombblockade transport via a tiny island, ${ }^{1}$ (ii) a reflectometry technique by connecting the SET to an impedance matching circuit composed of an inductor expands the readout bandwidth, and (iii) substantial reduction of low-frequency flicker noise due to the high operating frequency. However, in practice, the RF-SET is limited to use in only cryogenic sensing applications due to its reliance on Coulomb blockade. Silicon SETs with large charging energy can increase the working operating temperature; however, it is still in practice limited to less than $13 \mathrm{~K} .{ }^{9}$ The idea of the RF-SET has in recent years also been extended to charge sensors based on quantum point contacts (QPCs) in the form of the RF-QPC: ${ }^{10}$ however, like the RF-SET, the RF-QPC also requires cryogenic temperatures.

Silicon field-effect transistors (FETs) with nanometerscale channels represent a unique room-temperature charge detector that also achieves charge sensitivity with single-electron resolution. ${ }^{11}$ The small channel allows current characteristics to be modulated sensitively by a single electron ${ }^{11}$ and has the smaller Flicker noise in comparison with wide channels, ${ }^{12}$ leading to a charge sensitivity of $1.3 \times 10^{-3} \mathrm{e} / \mathrm{Hz}^{0.5}$ even at room temperature. Such features of the silicon nanometerscale FET expand the range of detectable charge signals and

${ }^{\text {a)} E-m a i l: ~ n i s h i g u c h i . k a t s u h i k o @ 1 a b . n t t . c o . j p a ~}$ have been recently used to sense infrared radiation, ${ }^{13}$ molecules, ${ }^{14}$ and mechanical motion. ${ }^{15}$ However, the operation speed of the dc FET is slow $(\sim 2 \mathrm{kHz})$ due to the large channel resistance $R_{\mathrm{C}}$ combined with the large stray capacitance $C_{\mathrm{S}}$ at the drain contact, giving a large $R_{\mathrm{C}} C_{\mathrm{S}}$ time. Additionally, the flicker noise at low frequency necessitates heavy filtering to achieve low noise, which also limits operation speed.

Here, we introduce a reflectometry technique using a nanometer-scale silicon FET incorporated with an LC tank circuit, which is referred to as an RF-FET. We use the tank circuit to match the low impendence of the RF electronics to the high-impedance of the nanometer sized FET channel. The nano-FET has capability of single-electron detection at room temperature and allows us to use a high-power carrier signal for reflectometry. Combining these two features, we achieve a combination of a room-temperature charge sensitivity of $2 \times 10^{-4} \mathrm{e} / \mathrm{Hz}^{0.5}$ with a $20 \mathrm{MHz}$ readout bandwidth.

Figures 1(a)-1(c) show the schematics for DC measurements of a FET-based electrometer (E-FET) used to detect a single electron. The detailed fabrication and mechanism are explained elsewhere. ${ }^{11}$ A storage node $(\mathrm{SN})$ is located close to the channel of the E-FET and connected to another transistor (T-FET) which transfers single electrons. The nanometer-scale E-FET's channel as well as a narrow gap between the channel and SN enable single electrons in the $\mathrm{SN}$ to modulate the current $I_{\mathrm{E}}$ flowing through the E-FET due to capacitive coupling between the SN and E-FET's channel. Additionally, the nanometer-scale FET reduces the Flicker noise. ${ }^{12}$ As a result, the motion of single electrons entering and leaving the SN can be monitored as a quantized change in $I_{\mathrm{E}}$ even at room temperature as shown in Fig. 1(e). Using this quantized change in $I_{\mathrm{E}}$ and transconductance of the E-FET, we calibrate the signal level corresponding to a single-electron sensitivity in the E-FET. ${ }^{11}$

In Fig. 2, we introduce the RF-FET. An equivalent circuit of the RF-FET is shown in Fig. 2(a). The E-FET is connected to an inductor $L$ of $431 \mathrm{nH}$. The inductor is connected in parallel with a stray capacitor $C_{\mathrm{S}}$ of $5.2 \mathrm{pF}$ originating from the E-FET and wiring. In order to characterize the 


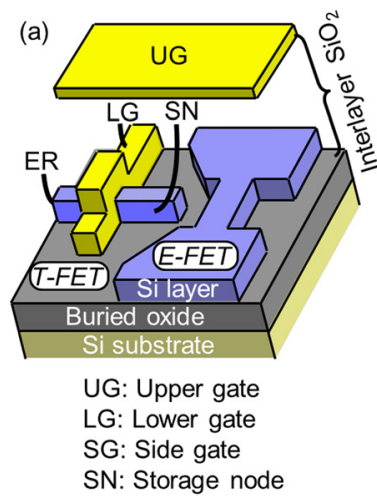

(b)
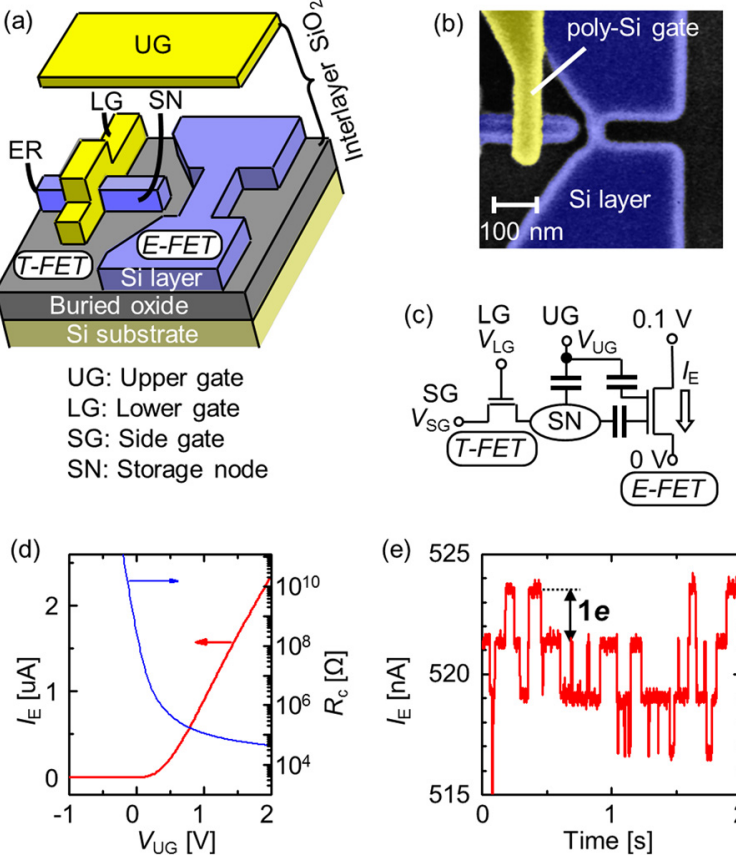

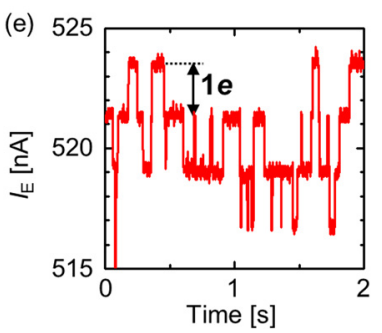

FIG. 1. Calibration of the single-electron signal level using a dc FET electrometer. (a) A birds-eye view. The upper gate (UG) convers the entire area. The device is oxidized after a lithographic patterning and etching. ${ }^{11}$ The device is estimated to have following dimensions after oxidization: the width and length of the E-FET channel are estimated to be 10 and $100 \mathrm{~nm}$, respectively; the channel width and gate length of the T-FET are 10 and $20 \mathrm{~nm}$, respectively; the gap between the $\mathrm{SN}$ and E-FET channel is $50 \mathrm{~nm}$. (b) A scanning electron microscope image without the UG. (c) Equivalent measurement circuit for $(\mathrm{d})$ current $\left(I_{\mathrm{E}}\right)$-voltage $\left(V_{\mathrm{UG}}\right)$ characteristics and (e) real-time single-electron detection. In (d), channel resistance $\left(R_{\mathrm{C}}\right)-V_{\mathrm{UG}}$ characteristics are also shown. $V_{\mathrm{LG}}$ and $V_{\mathrm{SG}}$ are 2 and $0 \mathrm{~V}$, respectively. In (e), $V_{\mathrm{UG}}, V_{\mathrm{LG}}$, and $V_{\mathrm{SG}}$ are $2,-2.35$, and $-1 \mathrm{~V}$, respectively. Since the $\mathrm{SN}$ is completely filled with electrons $(\sim 100 e)$, the potential energy of the $\mathrm{SN}$ is the same as that of the SG, and thus single electrons shuttle between the SN and SG. Each quantized change in $I_{\mathrm{E}}$ caused by the single-electron shuttling is constant. Measurements are performed with an Agilent 4156C at room temperature. For simplicity, in Figures 2-4, the combination of the T-FET and E-FET is equivalently expressed by only the E-FET: the T-FET is turned on using $V_{\mathrm{LG}}$ of $2 \mathrm{~V}$.

resonance frequency of the resulting LC tank circuit, measurements of the reflection coefficient $\mathrm{S} 11$ of the RF-FET are performed with a vector network analyzer. Figure 2(b) shows S11 characteristics of the RF-FET as a function of frequency. A sharp dip at a resonance frequency $f_{\text {reso }}$ of $106 \mathrm{MHz}$ is observed with a quality factor $\mathrm{Q}=3.03$. Using the measured resonance frequency of the RF-FET together with the known self-resonant frequency of the inductor $(550 \mathrm{MHz})$, we determine the total stray capacitance of the RF-FET configuration to be $5.2 \mathrm{pF}$, most of which arises from the capacitance of the drain pad to the substrate and from wiring between the FET and inductor.

In Fig. 2(b), we demonstrate the operation principle of the RF-FET. In the RF-FET, a voltage applied to the upper gate (UG) changes the channel resistance and, consequently, changes the sharpness and depth of S11characteritics at $f_{\text {reso }}$. This modulation of S11 characteristics is essential for the reflectometry technique: a target signal to be detected by the RF-FET is monitored as the change in $\mathrm{S} 11$ caused by the modulation of $R_{\mathrm{C}}$. To maximize the charge sensitivity, the dip in S11 at the resonant frequency should be as large and as sharp as possible. Theoretically, this is achieved by
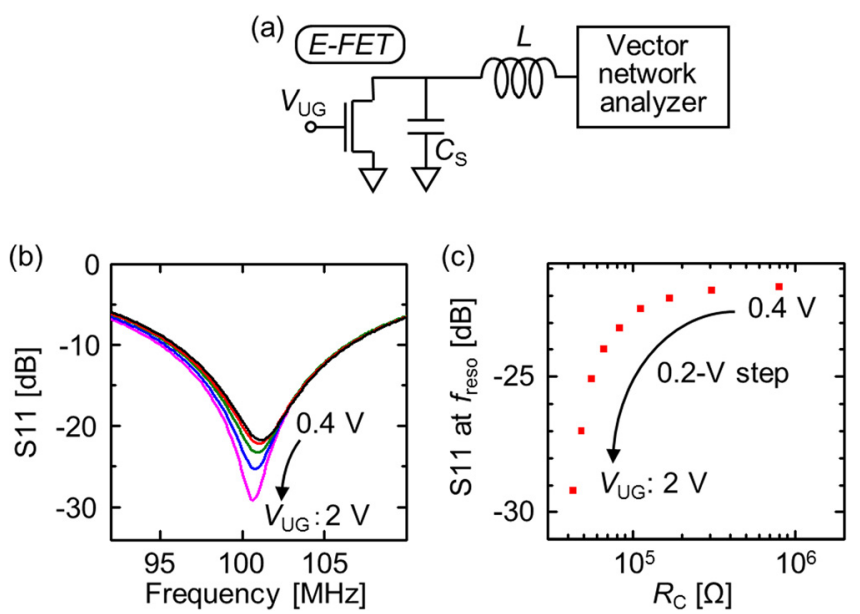

FIG. 2. Reflection coefficient S11 characteristics. (a) Measurement schematics of the RF-FET with inductor $L=431 \mathrm{nH}$. The stray capacitor $C_{\mathrm{S}}$ originating from the E-FET and wiring is evaluated to be $5.2 \mathrm{pF}$ from S11 characteristics. The vector network analyzer is Rohde \& Schwarz ZNB. (b) $\mathrm{S} 11$ characteristics when $V_{\mathrm{UG}}$ is changed in $0.4 \mathrm{~V}$ steps. The resonant frequency of the tank circuit is $106 \mathrm{MHz}$. (c) Change in $\mathrm{S} 11$ at $f_{\text {reso }}$ when $R_{\mathrm{C}}$ is changed by $V_{\mathrm{UG}}$. In (b), (c), $V_{\mathrm{UG}}$ is changed in an empirical range so as to suppress damage to the E-FET.

making the channel resistance $R_{\mathrm{C}}$ as close as possible to $L / C_{\mathrm{S}} Z_{0}$, where $Z_{0}$ is the characteristic impedance of the $\mathrm{RF}$ electronics $\left(Z_{0}=50 \Omega\right){ }^{5,16,17}$ Figure 2(c) shows the change in $\mathrm{S} 11$ at $f_{\text {reso }}$ when $R_{\mathrm{C}}$ is changed by tuning the UG voltage $V_{\mathrm{UG}}$. When $R_{\mathrm{C}}$ is reduced and thus becomes closer to $L /$ $C_{\mathrm{S}} Z_{0}$, the dip in $\mathrm{S} 11$ becomes deeper, as expected. In the following, we operate the RF-FET at a gate voltage $V_{\mathrm{UG}}=2 \mathrm{~V}$, corresponding to a channel resistance $R_{\mathrm{C}}=40 \mathrm{k} \Omega$, in order to optimize the sensitivity. Note that it is possible to obtain a deeper dip in S11 at larger channel resistances by reducing the stray capacitance Cs, which could be achieved in future devices by reducing the capacitance of the drain pad to the substrate.

In Fig. 3, we demonstrate detection of a high-frequency signal applied to the gate using reflectometry of the RF-FET. Schematics of the reflectometry setup using the RF-FET is shown in Fig. 3(a). Since the T-FET is turned on using $\mathrm{V}_{\mathrm{LG}}$ of $2 \mathrm{~V}$, the side gate (SG) is electrically connected to the SN. An RF carrier signal $S_{\text {carrier }}$ whose frequency $f_{\text {carrier }}$ is close to $f_{\text {reso }}$ is applied to the RF-FET. $f_{\text {carrier }}$ is adjusted so that the slope of $\mathrm{S} 11$ characteristics [see Fig. 2(b)] at $f_{\text {carrier }}$ is the steepest, which leads to the highest charge sensitivity. A target signal $S_{\text {target }}$ to be detected is then applied to the SG via the on-state T-FET, which is tuned here using its gate to act as a conducting channel. A frequency spectrum of a reflected signal $S_{\text {ref }}$ is then monitored with a spectrum analyzer. In order to evaluate the charge sensitivity precisely, the amplitude of $S_{\text {target }}$ applied to the SG is adjusted to correspond to the change in SN voltage caused by a single electron, as calibrated using the dc measurements, i.e., the change in $I_{\mathrm{E}}$ caused by a single electron and the transconductance of the E-FET (Fig. 1). ${ }^{11,18}$ Figure 3(b) shows the frequency spectrum of $S_{\text {ref }}$ when $S_{\text {target }}$ with frequency $f_{\text {target }}$ of $10 \mathrm{MHz}$ is applied to the SG. In $S_{\text {ref }}$ from the RF-FET, two peaks in addition to $S_{\text {carrier }}$ appear. These side peaks arise from mixing of $S_{\text {carrier }}$ applied at the drain of the nano-FET with $S_{\text {target }}$ applied to the SG. The distance between the main peak and 
(a)
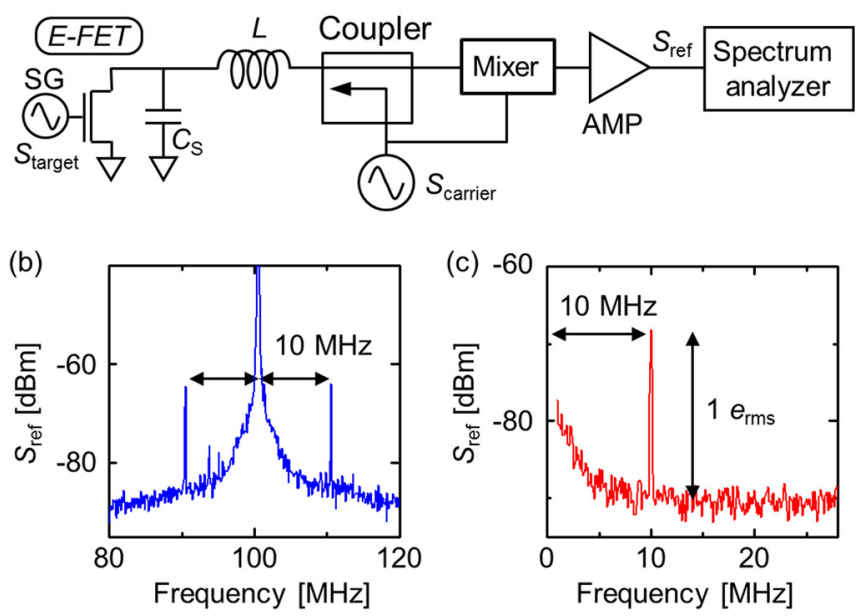

FIG. 3. Reflectometry technique using the RF-FET. (a) Measurement schematics. A carrier signal $S_{\text {carrier }}$ is applied to the RF-FET through a coupler. A target signal to be detected is applied to the SG. A reflected signal $S_{\text {ref }}$ is monitored by a spectrum analyzer after signal amplification of $28 \mathrm{~dB}$. $L$ and $C_{\mathrm{S}}$ are $431 \mathrm{nH}$ and $5.2 \mathrm{pF}$, respectively. The spectrum analyzer is a Rohde \& Schwarz FSL. Signal generators for $S_{\text {carrier }}$ and $S_{\text {target }}$ are Rohde \& Schwarz SMB 100A. Frequency spectra of $S_{\text {ref }}$ (b) without and (c) with a mixer. The carrier signal has a power of $10 \mathrm{dBm}$ and a frequency of $100 \mathrm{MHz}$. Power of $S_{\text {target }}$ is $-28 \mathrm{dBm}$, which corresponds to a signal level of $1 e(\mathrm{rms})$. The resolution bandwidth is $100 \mathrm{kHz}$.

each side peak corresponds to $f_{\text {target }}$, allowing us to identify $S_{\text {target }}$ as the origin of the side-band peaks. ${ }^{7,9,10}$

In order to quantitatively asses the charge sensitivity of the RF-FET, we feed $S_{\text {ref }}$ from the RF-FET into a mixer, together

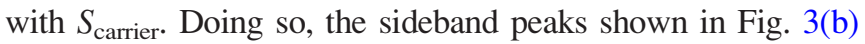
are down-mixed to a single peak in the output of the mixer at the same frequency as $S_{\text {target }}$ applied to the SG. The spectrum of the resulting signal out of the mixer is shown in Figure 3(c). Note that the noise floor comes from the spectrum analyzer and not the intrinsic noise of the RF-FET. $S_{\text {target }}$ corresponding to a single electron is detected with a signal-to-noise ratio (SNR) of $21 \mathrm{~dB}$ and charge sensitivity of $2 \times 10^{-4} \mathrm{e} / \mathrm{Hz}^{0.5}$, which is given by $e /(2 \times \mathrm{RBW})^{-0.5} 10^{-\mathrm{SNR} / 20}$, where the factor 2 accounts for the power collected from both side bands, and $\mathrm{RBW}$ is the resolution bandwidth of the spectrum analyzer configured during the measurements $(100 \mathrm{kHz})$.

In Fig. 4, we evaluate the charge sensitivity as a function of the power $P_{\text {carrier }}$ of $S_{\text {carrier. }}$ In principle, increasing the amplitude of $S_{\text {carrier }}$ will result in a larger amplitude of the
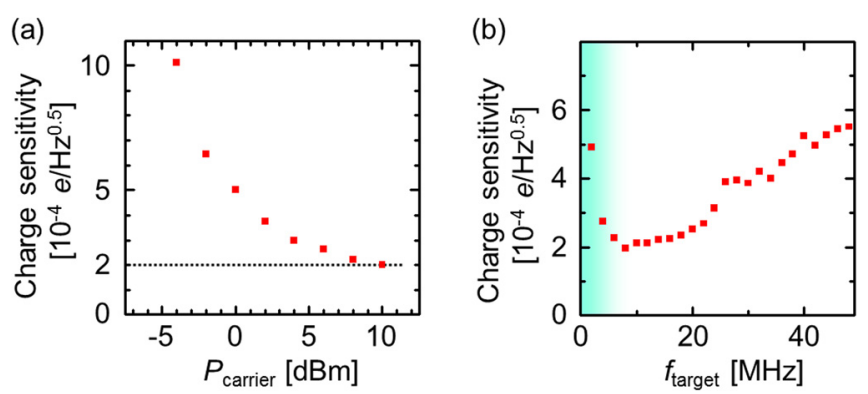

FIG. 4. Charge sensitivity characteristics of the RF-FET. (a) Dependence of the charge sensitivity on power $P_{\text {carrier }}$ with $P_{\text {target }}=-28 \mathrm{dBm}$ and $f_{\text {target }}=10 \mathrm{MHz}$. (b) Dependence of charge sensitivity on $f_{\text {target }}$ with $P_{\text {carrier }}=10 \mathrm{dBm}$ and $P_{\text {target }}=-28 \mathrm{dBm}$. The shaded area represents the frequency range in which the noise floor originating from the spectrum analyzer is increased at low frequencies, as shown in Fig. 3(c). charge-detection signal. In RF-SET devices, the maximum $P_{\text {carrier }}$ is limited by the small charging energy of the SET: further increases of the amplitude of $S_{\text {carrier }}$ do not increase the charge signal due to vanishing transconductance of the SET. A significant advantage of the RF-FET is that the transconductance is linear up to much higher source-drain voltages, allowing significantly larger $P_{\text {carrier }}$ to be used. In Fig. 4(a), we show the change in the charge sensitivity of the RFFET as a function of $P_{\text {carrier }}$ Increasing $P_{\text {carrier, }}$, the charge sensitivity is improved as expected. In Fig. 4(a), the improvement of the charge sensitivity shows a tendency to saturate at $P_{\text {carrier }}$ larger than $10 \mathrm{dBm}$ because large $P_{\text {carrier }}$ increases the noise coming from the FET, degrades mobility of the FET, and puts the FET in a pinch-off condition. Note this saturation power corresponding to a AC voltage of $707 \mathrm{mV}$. This is considerably higher than the maximum useful AC source-drain voltage for an RF-SET, which is limited to the charging energy of the SET, typically in the range of a few $\mathrm{mVs}$.

In Fig. 4(b), we present data demonstrating the large readout bandwidth of the RF-FET. Fig. 4(b) shows the dependence of the charge sensitivity on frequency $f_{\text {target }}$ of $S_{\text {target }}$. We achieve a charge sensitivity of $\sim 2 \times 10^{-4} \mathrm{e} / \mathrm{Hz}^{0.5}$ in a range of $f_{\text {target }}$ between 8 and $20 \mathrm{MHz}$. When $f_{\text {target }}$ is lower than $6 \mathrm{MHz}$ (shaded area), the charge sensitivity becomes worse due to large noise floor originating from the spectrum analyzer [shown in Fig. 3(c)]. At high frequencies, the charge sensitivity is gradually degraded due to the bandwidth limitation of the tank circuit, given by $f_{\text {reso }} / Q=35 \mathrm{MHz}$, where $Q$ is the quality factor of S11 characteristics [Fig. 2(b)]. ${ }^{10,16,17}$ In reflectometry experiments with tank circuits, higher $\mathrm{Q}$ is, in general, desirable to obtain a higher charge sensitivity. However, this higher $Q$ also leads to a reduced detector bandwidth. By taking advantage of the large $P_{\text {carrier }}$ possible with the RF-FET, a high charge sensitivity can be achieved even a relatively small Q of 3. Doing so, we are able to achieve a combination of a high charge sensitivity of $2-4 \times 10^{-4} \mathrm{e} / \mathrm{Hz}$ with a bandwidth of $30 \mathrm{MHz}$. Using the RF-FET, we have both enhanced the charge sensitivity and simultaneously expanded the bandwidth of charge detection with the nanoscale FET by more than two orders of magnitude.

Another noteworthy feature of the RF-FET using a combination of the T-FET and E-FET is a precise evaluation of the charge sensitivity. Since the charge sensitivity is extremely small, its evaluation needs a standard signal with high precision, which is, typically, a single electron. In other reports using RF-SETs, current characteristics originating from the Coulomb blockade are used to evaluate the standard signal corresponding to a single electron. However, as it is extremely difficult to achieve Coulomb blockade at room temperature, another way to evaluate the standard signal is required. The combination of the T-FET and E-FET which allows single-electron detection at room temperature as shown in Fig. 1(e) plays an important role in the evaluation of the standard signal.

Finally, we discuss the ideal charge sensitivity in terms of the fundamental noise limits in the RF-FET. As shown in Fig. $3(\mathrm{~b}), S_{\text {target }}$ is monitored using a reflection signal at $f_{\text {carrier }}-f_{\text {target }}$. Therefore, the charge sensitivity or SNR is dominated by the noise in the frequency range of the readout bandwidth centered 
at $f_{\text {carrier}}$, which substantively eliminates flicker noise at low frequency. The noise in an FET at high frequency originates from the Johnson noise, which is given by $-174+10$ $\log (\mathrm{RBW})=-124 \mathrm{dBm}$ at room temperature. Since the gain of the amplifier is $28 \mathrm{~dB}$, the theoretical noise floor originating from the RF-FET is expected to be $-96 \mathrm{dBm}$, which is lower than the noise floor $(-91 \mathrm{dBm})$ of the spectrum analyzer in our measurements. A larger amplifier gain could increase the SNR by $5 \mathrm{~dB}$, resulting in a further improvement of the charge sensitivity to $1.1 \times 10^{-4} \mathrm{e} / \mathrm{Hz}^{0.5}$.

In summary, we have demonstrated a reflectometry technique using a small FET at room temperature. Using an FET that is optimized to detect single electrons and taking advantage of the high power carrier signal possible with the FET, a combination of a charge sensitivity of $2 \times 10^{-4} \mathrm{e} / \mathrm{Hz}^{0.5}$ with a bandwidth of $30 \mathrm{MHz}$ is obtained at room temperature. The high-sensitivity and large bandwidth of the RF-FET, not before possible outside of cryogenic experiments, will open up applications such as detecting tiny charge signals from devices such as nano-mechanical sensors.

We are grateful to H. Inokawa of Shizuoka University for valuable discussions about the reflectometry technique. We also thank I. Mahboob and D. Hatanaka of NTT and A. Castellanos-Gomez, V. Singh, S. J. Bosman, B. H. Schneider, and H. B. Meerwaldt of Delft University of Technology for experimental support and helpful discussions. This work was partly supported by the Funding
Program for Next Generation World-Leading Researchers of JSPS (GR103).

${ }^{1}$ M. H. Devoret and R. J. Schoelkopf, Nature 406, 1039 (2000).

${ }^{2}$ J. Hahm and C. M. Lieber, Nano Lett. 4, 51 (2004).

${ }^{3}$ A. Fujiwara and Y. Takahashi, Nature 410, 560 (2001).

${ }^{4}$ R. G. Knobel and A. N. Cleland, Nature 424, 291 (2003).

${ }^{5}$ R. J. Schoelkopf, P. Wahlgren, A. A. Kozhevnikov, P. Delsing, and D. E. Prober, Science 280, 1238 (1998).

${ }^{6}$ A. Aassime, G. Johansson, G. Wendin, R. J. Schoelkopf, and P. Delsing, Phys. Rev. Lett. 86, 3376 (2001).

${ }^{7}$ W. Lu, Z. Ji, L. Pfeiffer, K. W. West, and A. J. Rimberg, Nature 423, 422 (2003).

${ }^{8}$ T. Fujisawa, T. Hayashi, R. Tomiya, and Y. Hirayama, Science 312, 1634 (2006).

${ }^{9}$ M. Manoharan, Y. Tsuchiya, S. Oda, and H. Mizuta, Nano Lett. 8, 4648 (2008).

${ }^{10}$ D. J. Reilly, C. M. Marcus, M. P. Hanson, and A. C. Gossard, Appl. Phys. Lett. 91, 162101 (2007).

${ }^{11}$ K. Nishiguchi, C. Koechlin, Y. Ono, A. Fujiwara, H. Inokawa, and H. Yamaguchi, Jpn. J. Appl. Phys. 47, 8305 (2008).

${ }^{12}$ N. Clement, K. Nishiguchi, A. Fujiwara, and D. Vuillaume, Nat. Commun. 1, 92 (2010).

${ }^{13}$ K. Nishiguchi, A. Fujiwara, Y. Ono, H. Inokawa, and Y. Takahashi, Appl. Phys. Lett. 90, 223108 (2007).

${ }^{14}$ K. Nishiguchi, N. Clement, T. Yamaguchi, and A. Fujiwara, Appl. Phys. Lett. 94, 163106 (2009).

${ }^{15}$ I. Mahboob, E. Flurin, K. Nishiguchi, A. Fujiwara, and H. Yamaguchi, Appl. Phys. Lett. 97, 253105 (2010).

${ }^{16}$ A. N. Korotkov and M. A. Paalanen, Appl. Phys. Lett. 74, 4052 (1999).

${ }^{17}$ L. Roschier, P. Hakonen, K. Bladh, P. Delsing, K. W. Lehnert, L. Spietz, and R. J. Shoelkopf, J. Appl. Phys. 95, 1274 (2004).

${ }^{18}$ K. Nishiguchi, H. Inokawa, Y. Ono, A. Fujiwara, and Y. Takahashi, Electron. Lett. 40, 229 (2004). 